\section{Toothbrush handles created from plant waste}

In October, Avient announced the launch of new bio-filled polymer grades at Fakuma 2021, the international trade show for plastics processing.

The new reSound NF bio-filled grades are based on polymers such as polypropylene (PP) with $15-20 \%$ bio-based filler. The filler is sourced from plant waste that would otherwise be landfilled, which in turn would create methane, a greenhouse gas more potent than carbon dioxide.

The new materials have a pleasing aesthetic compared to alternative natural fibre-filled polymer grades, are fully colourable, and can be formulated to meet various regulatory compliance standards, making them suitable for consumer applications such as household items and personal care products.

One early adopter of the new materials is Turkish toothbrush brand, Difaş. Looking to differentiate in the market and meet the desires of consumers, Difaş worked with Avient to develop a solution for toothbrush handles, combs and hairbrush handles that

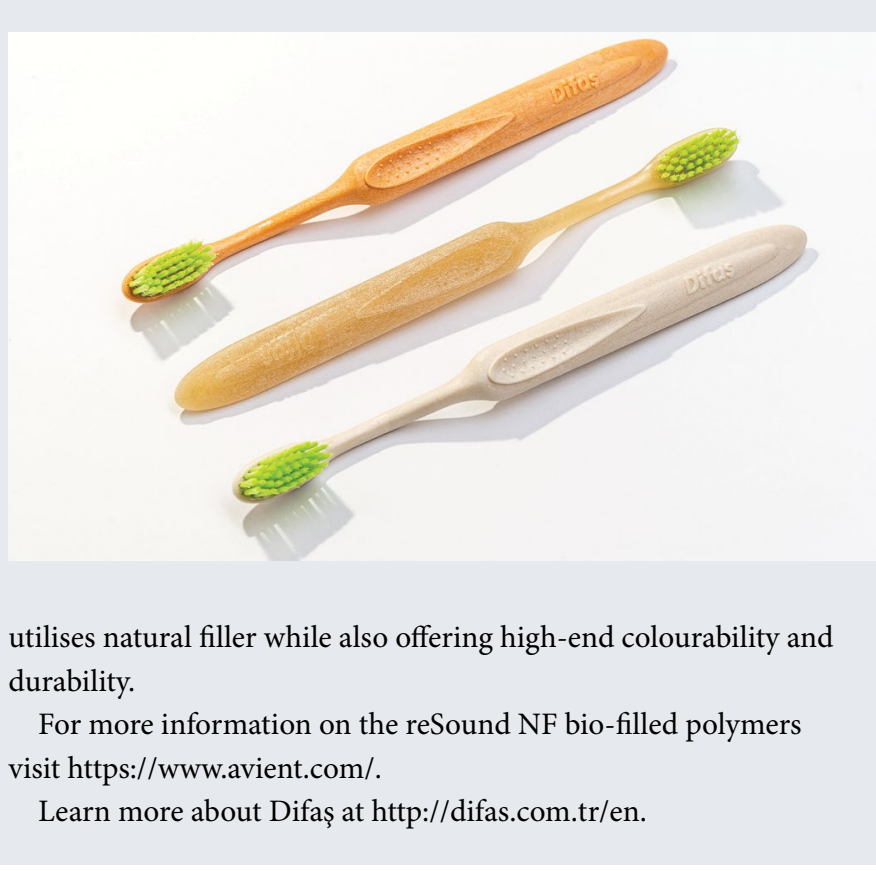

\section{New in-practice trial kit}

Whether at the start of an improved oral hygiene journey or looking for ways to upgrade from a manual approach, professional recommendation is a significant trigger for millions of dental patients. However, professionals need the right tools to be able to offer their patients a proactive as opposed to a reactive approach to transitioning to a powered brush. To help with this goal, Philips has introduced an in-practice Sonicare trial unit which allows patients to experience the unique sonic toothbrush feeling, risk-free, as part of their appointment and take away a free brush head.

The new in-practice trial kit includes everything dental professionals need to help patients experience a new level of clean, coach them and address possible concerns they may have - and all this without the pressure of the patient having to make a purchase there and then.

The Sonicare starter kit which contains one ProtectiveClean 4700 power handle, 60 premium brush heads ( $30 \mathrm{x}$ plaque and $30 \mathrm{x}$ gum), 60 handle sleeves, 60 brush head bags and one charger base, along with disinfection advice. This provides dental professionals with a tool to allow them to initiate a proactive conversation about manual-to-power toothbrush conversion.

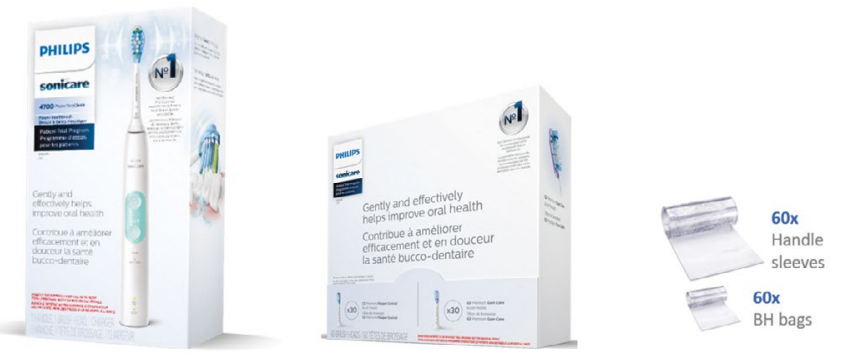

The Sonicare ProtectiveClean toothbrush features all the core elements which make the Sonicare brush so effective - but one of its primary attractions is its variable intensity settings which makes it so appealing for patients transitioning from a manual toothbrush. This allows dental professionals to show patients how to tune it up or down as they get used to the sonic sensation and to customise their brushing experience. The slim handle design is also lightweight and easy to manoeuvre for patients who may only have used a manual brush. All Philips Sonicare brush heads are also recyclable through the company's Terracycle scheme.

To find out more about the in practice trial kit and to sign up, contact dental.sales.support@philips.com.

\title{
New precision milling centre opened in Sweden
}

On 7 October 2021, friends and colleagues of Neoss Ltd. and ARC Solutions AB came together to celebrate the opening of a new stateof-the-art facility; the new ARC Solutions milling centre.

Since the acquirement of ARC Solutions in 2018, Neoss's bid to expand the offering of digital implant solutions has gone from strength to strength, seeing ARC produce thousands of high-quality advanced customised implant-retained prosthetics and abutments, resulting in quickly outgrowing their former facilities.

Dr Robert Gottlander, President and CEO of Neoss, said: 'With the high demand of advanced customised implant-retained prosthetics and advanced engineering solutions, we want to offer more of ARC's products and services to our customers. ARC's advanced customised implant-retained prosthetics and advanced engineering solutions are a perfect fit with our leading Neoss ProActive dental implant offerings'.

The new centre will allow the team to be much more efficient, and facilitates the room for new equipment, allowing the company to produce precision milled dental implant constructions, focusing on quality and reliability.

To discover more about ARC Solutions, visit: https://www. arcsolutions.se. 